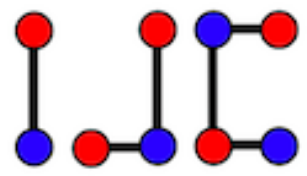

\title{
Further results on locating chromatic number for amalgamation of stars linking by one path
}

\author{
Asmiati $^{\mathrm{a}}$, L. Yulianti ${ }^{\mathrm{b}}$, C. Ike Tri Widyastuti ${ }^{\mathrm{c}}$ \\ ${ }^{a}$ Department of Mathematics, Lampung University, Bandar Lampung, Indonesia \\ ${ }^{b}$ Department of Mathematics, Andalas University, Limau Manis Padang, Indonesia \\ ${ }^{c}$ Fransiskus Senior High School, Bandar Lampung, Indonesia \\ asmiati.1976@fmipa.unila.ac.id, lyra@sci.unand.ac.id,ike_widya76@yahoo.com
}

\begin{abstract}
Let $G=(V, E)$ be a connected graph. Let $c$ be a proper coloring using $k$ colors, namely $1,2, \cdots, k$. Let $\Pi=\left\{S_{1}, S_{2}, \cdots, S_{k}\right\}$ be a partition of $V(G)$ induced by $c$ and let $S_{i}$ be the color class that receives the color $i$. The color code, $c_{\Pi}(v)=\left(d\left(v, S_{1}\right), d\left(v, S_{2}\right), \cdots, d\left(v, S_{k}\right)\right)$, where $d\left(v, S_{i}\right)=\min \left\{d(v, x) \mid x \in S_{i}\right\}$ for $i \in[1, k]$. If all vertices in $V(G)$ have different color codes, then $c$ is called as the locating-chromatic $k$-coloring of $G$. Minimum $k$ such that $G$ has the locating-chromatic $k$-coloring is called the locating-chromatic number, denoted by $\chi_{L}(G)$. In this paper, we discuss the locating-chromatic number for $n$ certain amalgamation of stars linking a path, denoted by $n S_{k, m}$, for $n \geq 1, m \geq 2, k \geq 3$, and $k>m$.
\end{abstract}

\section{Introduction}

The locating chromatic number is a topic in graph theory, derived from the vertex-coloring and partition dimension of a graph [11]. Many paper discussed about the locating chromatic number since Chartrand et al. [9] introduced the concept in 2002.

All graphs considered are finite, undirected and simple. Let $G=(V, E)$ be a connected graph. Let $c$ be a proper coloring using $k$ colors, namely $1,2, \cdots, k$. Let $\Pi=\left\{S_{1}, S_{2}, \cdots, S_{k}\right\}$ be a partition of $V(G)$ induced by $c$ and let $S_{i}$ be the color class that receives the color $i$. The

Received: 01 Feb 2018, Revised: 01 Jun 2018, Accepted: 08 Jun 2018. 
color code, $c_{\Pi}(v)=\left(d\left(v, S_{1}\right), d\left(v, S_{2}\right), \cdots, d\left(v, S_{k}\right)\right)$, where $d\left(v, S_{i}\right)=\min \left\{d(v, x) \mid x \in S_{i}\right\}$ for $i \in[1, k]$. If all vertices in $V(G)$ have different color codes, then $c$ is called as the locatingchromatic $k$-coloring of $G$. Minimum $k$ such that $G$ has the locating-chromatic $k$-coloring is called the locating-chromatic number, denoted by $\chi_{L}(G)$.

Theorem 1.1. [10] Let $G$ be a simple connected graph and $c$ be a locating coloring of $G$. If $v, w \in V(G)$ and $v \neq w$ such that $d(v, x)=d(w, x)$ for all $x \in V(G)-\{v, w\}$, then $c(v) \neq c(w)$. In particular, if $v$ and $w$ are non adjacent vertices of $G$ such that neighborhood of $v$ is equal to neighborhood of $w$, then $c(v) \neq c(w)$.

Corollary 1.1. [10] If $G$ is a simple connected graph containing a vertex that is adjacent to $k$ leaves of $G$, then $\chi_{L}(G) \geq k+1$.

Chartrand et al. [9][10] obtained the locating chromatic number of some classes of graphs such that: paths, stars, double stars, caterpillars, complete graphs, bipartite graphs, and the characterization of graphs having locating chromatic number $n,(n-1)$, or $(n-2)$. Next, Asmiati et al. investigated locating chromatic number for special kind of trees, namely: amalgamation of stars [1], [4], firecracker graphs [2], banana trees [5]. Moreover, Baskoro at al. [8] determined the locating chromatic number for corona product of some graphs. Beside that, Asmiati et al. [3] characterized graphs containing cycle having locating chromatic number tree and Baskoro et al. [7] characterized all trees having locating chromatic number three.

Let $S_{m+2}$ be a star with $(m+2)$ vertices. The amalgamation of stars, denoted by $S_{k, m}$, where $k, m \geq 2$, is obtained from $(k-1)$ stars $S_{m+2}$, by identifying one leaf of every stars $S_{m+2}$. The identified vertex is denoted as the center of $S_{k, m}$. Graph $n S_{k, m}$ is obtained from $n$ copies $S_{k, m}$ and every center of them, denoted by $x_{i}$, for $i=1,2, \ldots, n$ is linked by one path, and $(n-1)$ new vertices denoted $y_{i}, i=1,2, \ldots, n-1$ are the subdivision vertices in $x_{i} x_{i+1}, i=1,2, \ldots, n-1$. Next, the vertices of distance 1 from the center $x_{i}$ are defined as the intermediate vertices, denoted by $l_{j}^{i}, i=1,2, \ldots, n, j=1,2, \ldots, k-1$ and the $t$-th leaf of the intermediate vertices $l_{j}^{i}$ are denoted by $l_{j t}^{i}(t=1,2, \ldots, m)$.

In [6], Asmiati et al. determined the locating chromatic number of $n S_{k, m}$ for $k \leq m$, where $k \geq 3$ and $m \geq 2$, as follows.

$$
\chi_{L}\left(n S_{k, m}\right)= \begin{cases}m+1, & 1 \leq n \leq\left\lfloor\frac{m}{k-1}\right\rfloor \\ m+2, & \text { otherwise }\end{cases}
$$

In this paper we will discuss the locating chromatic number of $n S_{k, m}$ for $k>m$, where $k \geq 3$ and $m \geq 2$.

\section{Main Results}

In this section, we will discuss about the locating chromatic number of $n S_{k, m}$ for $n \geq 1$ and $k>m, k \geq 3, m \geq 2$.

Lemma 2.1. Let c be a coloring on $n S_{k, m}$ using $(k-a)$ colors, where $k>m, k \geq 3, m \geq 2, a \geq$ $0, a=k-m-1$. Coloring $c$ is a locating coloring if and only if $c\left(l_{j}^{i}\right)=c\left(l_{n}^{s}\right), j \neq n$ and $i \neq s$ such that $\left\{c\left(l_{j t}^{i}\right) \mid t=1,2,3, \ldots, m\right\}$ and $\left\{c\left(l_{n t}^{s}\right) \mid t=1,2,3, \ldots, m\right\}$ are two different sets. 
Proof. Consider $P=\left\{c\left(l_{j t}^{i}\right) \mid t=1,2,3, \ldots, m\right\}$ and $Q=\left\{c\left(l_{n t}^{s}\right) \mid t=1,2,3, \ldots, m\right\}$. Let $c$ be a locating coloring of $n S_{k, m}, k>m, k \geq 3, m \geq 2, a \geq 0$, dan $c\left(l_{j}^{i}\right)=c\left(l_{n}^{s}\right)$, for some $j \neq n$, and $i \neq s$. Suppose that $P=Q$. Since $d\left(l_{j}^{i}, u\right)=d\left(l_{n}^{s}, u\right)$ for each $u \in V \backslash\left\{\left\{l_{j t}^{i}\right\} \cup\left\{l_{n k}^{s}\right\}\right\}$, then the color codes of $l_{j}^{i}$ and $l_{n}^{s}$ are the same. So, $c$ is not a locating coloring, a contrary. As the result, $P \neq Q$.

Let $\Pi$ be a partition of $V(G)$ with $|\Pi| \geq m$. Consider $c\left(l_{j}^{i}\right)=c\left(l_{n}^{s}\right), j \neq n$, dan $i \neq s$. Since $P \neq Q$, then there are two colors, namely $x$ and $y$ such that $(x \in P, x \notin Q)$ or $(y \in P, y \notin Q)$. Next, we will show that every $v \in V\left(n S_{k, m}\right)$ have different color codes.

- It is clear that $c_{\Pi}\left(l_{j}^{i}\right) \neq c_{\Pi}\left(l_{n}^{s}\right)$, since their color codes are different in the $x$-ordinat or $y$-ordinat.

- If $c\left(l_{j t}^{i}\right)=c\left(l_{n t}^{s}\right)$, for each $l_{j}^{i} \neq l_{n}^{s}$, then we divide two cases to show that $c_{\Pi}\left(l_{j t}^{i}\right) \neq c_{\Pi}\left(l_{n t}^{s}\right)$ Case 1: If $c\left(l_{j t}^{i}\right)=c\left(l_{n t}^{s}\right)$, then based on the previous proof $P \neq Q$. So, $c_{\Pi}\left(l_{j t}^{i}\right) \neq c_{\Pi}\left(l_{n t}^{s}\right)$. Case 2: Consider $c\left(l_{j}^{i}\right)=p_{1}$ and $c\left(l_{n}^{s}\right)=p_{2}$, where $p_{1} \neq p_{2}$. Then $c_{\Pi}\left(l_{j t}^{i}\right) \neq c_{\Pi}\left(l_{n t}^{s}\right)$ because their color codes are different at least in the $p_{1}$-ordinat and $p_{2}$-ordinat.

- If $c\left(x_{i}\right)=c\left(l_{j t}^{i}\right)$, then the color code of $c_{\Pi}\left(x_{i}\right)$ contains at least two components with value 1 , whereas in $c_{\Pi}\left(l_{j t}^{i}\right)$ contains exactly one component with value 1 . So, $c_{\Pi}\left(x_{i}\right) \neq c_{\Pi}\left(l_{j t}^{i}\right)$.

- If $c\left(y_{i}\right)=c\left(l_{j t}^{i}\right)$, then the color code of $c_{\Pi}\left(y_{i}\right)$ contains at least two components with values 1. whereas in $c_{\Pi}\left(l_{j t}^{i}\right)$ contains exactly one component with value 1 . So, $c_{\Pi}\left(y_{i}\right) \neq c_{\Pi}\left(l_{j t}^{i}\right)$.

From all cases, we can see that all vertices in $n S_{k, m}$ have different color codes, so $c$ is a locating coloring.

Lemma 2.2. Let $n \geq 1, k>m, k \geq 3, m \geq 2, a \geq 0$, and $a=k-m-1$. If $c$ is a locating coloring of $n S_{k, m}$ using $k-$ a colors and $H(a)=\left[\frac{(k-a-1)\left(\begin{array}{c}k-a-1 \\ m\end{array}\right)}{k-1}\right\rfloor$, then $n \leq H(a)$.

Proof. Let $c$ be a $(k-a)$-locating coloring of $n S_{k, m}$. For some $j$, consider $c\left(l_{j}^{i}\right)$ as the color of $l_{j}^{i}$, then the color combination of $\left\{l_{j t}^{i} \mid t=1,2,3, \ldots, m\right\}$ is $\left(\begin{array}{c}k-a-1 \\ m\end{array}\right)$. Since one color has been used for the central vertex $x$, then there are $(k-a-1)$ colors left to be assigned to $l_{j}^{i}$, for each $i=1,2, \ldots, n$ and $j=1,2,3, \ldots, k-1$. By Lemma 2.1, the maximum number for $n$ is $\left\lfloor\frac{(k-a-1)\left(\begin{array}{c}k-a-1 \\ m\end{array}\right)}{k-1}\right\rfloor=H(a), a \geq 0$.

Theorem 2.1. Let $n S_{k, m}$ be some certain amalgamation of stars for $a \geq 0, k>m, k \geq 3, m \geq 2$, $a=k-m-1$. Then

$$
\chi_{L}\left(n S_{k, m}\right)= \begin{cases}k-a, & 1 \leq n \leq H(a) \\ k-a+1, & \text { otherwise }\end{cases}
$$


Proof. First, we determine the lower bound of $\chi_{L}\left(n S_{k, m}\right)$ for $1 \leq n \leq H(a)=\left\lfloor\frac{(k-a-1)\left(\begin{array}{c}k-a-1 \\ m\end{array}\right)}{k-1}\right\rfloor$. Since every vertex $l_{j}^{i}$ for $i=1,2,3, \ldots, n$ and $j=1,2,3, \ldots, k-1$ are adjacent to $m=k-a-1$ leaves, then by Corollary 1.1, we have $\chi_{L}\left(n S_{k, m}\right) \geq k-a$.

To determine the upper bound of $\chi_{L}\left(n S_{k, m}\right)$ for $1 \leq n \leq H(a)=\left\lfloor\frac{(k-a-1)\left(\begin{array}{c}k-a-1 \\ m\end{array}\right)}{k-1}\right\rfloor$, let $c$ be a coloring of $V\left(n S_{k, m}\right)$ using $(k-a)$ colors. We assign the coloring as follows.

- $c\left(x_{i}\right)=1$, for $i=1,2,3, \ldots, n$.

- $c\left(y_{i}\right)=2$, for odd $i$ and 3 for even $i=1,2,3, \ldots, n$.

- Color of $l_{i}^{j}$ for each $i=1,2, \ldots, n$ and $j=1,2, \ldots,(k-1)$ given color $2,3, \ldots,(k-a)$, respectively.

- $\left\{c\left(l_{j t}^{i}\right)\right\}=\{1,2,3, \ldots, k-a\} \backslash\left\{c\left(l_{i}^{j}\right)\right\}$ for $t=1,2,3, \ldots, m$.

Next, we will show that all vertices in $V\left(n S_{k, m}\right)$ have different color codes. Consider $u, v \in$ $V\left(n S_{k, m}\right)$ and $c(u)=c(v)$. Then we have the following cases.

- If $u=x_{i}, v=x_{k}$ for some $i, k$ and $i \neq k$, then $c_{\Pi}(u) \neq c_{\Pi}(v)$ because $c\left(l_{j}^{i}\right) \neq c\left(l_{j}^{k}\right)$ for each $i=1,2, \ldots,(k-1)$.

- If $u=x_{i}, v=l_{j t}^{h}$ for some $i, h, j, t$, then in $c_{\Pi}(u)$ does not have component value four, whereas in $c_{\Pi}(v)$, exactly one component has value 4 . So, $c_{\Pi}(u) \neq c_{\Pi}(v)$.

- If $u=y_{i}, v=l_{j}^{i}$, for some $i, j$, then in $c_{\Pi}(u)$ exactly two components have value 1 , whereas in $c(v)$, at least three components have value 1 . So, $c_{\Pi}(u) \neq c_{\Pi}(v)$.

- If $u=y_{i}, v=l_{j}^{k}$, for some $i, k, j$ and $i \neq k$, then in $c_{\Pi}(u)$ exactly two components have value 1 , whereas in $c(v)$, at least three components have value 1 . So, $c_{\Pi}(u) \neq c_{\Pi}(v)$.

- If $u=y_{i}, v=l_{j t}^{i}$ for some $i, j, t$, then in $c_{\Pi}(u)$, exactly two components have value 1 , whereas in $c(v)$, exactly one component has value 1 . As a result, $c_{\Pi}(u) \neq c_{\Pi}(v)$.

- If $u=y_{i}, v=l_{j t}^{k}$ for some $i, k, j, t$ and $i \neq k$, then in $c_{\Pi}(u)$ at least two components have value 1 , whereas in $c(v)$, exactly one component has value 1 . So, $c_{\Pi}(u) \neq c_{\Pi}(v)$.

- If $u=l_{j}^{i}, v=l_{j t}^{i}$ for some $i, j, t$, then in $c_{\Pi}(u)$ at least two components have value 1 , whereas in $c(v)$, exactly one component has value 1 . As a result, $c_{\Pi}(u) \neq c_{\Pi}(v)$

- If $u=l_{j}^{i}, v=l_{h t}^{k}$ for some $i, j, k, h, t$ and $i \neq k, j \neq h$, then in $c_{\Pi}(u)$, at least two components have value 1 , whereas in $c(v)$, exactly one component has value 1 . So, $c_{\Pi}(u) \neq$ $c_{\Pi}(v)$

- if $u=l_{j t}^{i}, v=l_{h t}^{i}$ for some $i, j, h, t, j \neq h$. Since $\left\{c\left(l_{j t}^{i}\right)\right\} \neq\left\{c\left(l_{h t}^{i}\right)\right\}$, then $c_{\Pi}(u) \neq c_{\Pi}(v)$. 
- If $u=l_{j t}^{i}, v=l_{j t}^{k}$ for some $i, j, k, t, i \neq k$. Since $c\left(l_{j}^{i}\right) \neq c\left(l_{j}^{k}\right)$, then $c_{\Pi}(u) \neq c_{\Pi}(v)$.

Since all vertices have different color codes, then $c$ is a locating coloring on $n S_{k, m}$. Thus, $\chi_{L}\left(n S_{k, m}\right) \leq$ $k-a$ for $n \leq H(a)$.

Next, we discuss the locating chromatic number of $n S_{k, m}$ for $n>H(a)$.

By Corollary 1.1, we have the trivial lower bound, $\chi_{L}\left(S_{k, m}\right) \geq k-a$ for $>H(a)$. Suppose that $c$ is a locating coloring using $(k-a)$ colors on $n S_{k, m}$ for $k>m, k \geq 3, m \geq 2$, and $n>$ $H(a)$. Since $n>H(a)$, then there are $i, j, k, t, i \neq k$ and $\left\{c\left(l_{j}^{i}\right), c\left(l_{j t}^{i}\right)\right\}=\left\{c\left(l_{j}^{k}\right), c\left(l_{j t}^{k}\right)\right\}=$ $\{1,2,3, \ldots, k-a\}$ such that $c_{\Pi}\left(l_{j}^{i}\right)=c_{\Pi}\left(l_{j}^{k}\right)$ for some $j=1,2,3, \ldots, k-1, t=1,2,3, \ldots, m$, a contrary. Thus, $\chi_{L}\left(S_{k, m}\right) \geq k-a+1$ for $n>H(a)$.

Let $c$ be a coloring on $n S_{k, m}$ using $(k-a+1)$ colors. We assign the coloring as follows.

- $c\left(x_{i}\right)=1$, for $i=1,2,3, \ldots, n$.

- $c\left(y_{i}\right)=2$, for odd $i$ and 3 for even $i=1,2,3, \ldots, n$.

- For $j=1,2,3, \ldots,(k-1), c\left(l_{i}^{j}\right)=2$, for odd $i$ and 3 for even $i=1,2,3, \ldots, n$.

- If $A=\{1,2, \ldots, k-a+1\}$, define:

$$
\left.\left\{c\left(l_{j t}^{i}\right) \mid t=1,2, \ldots, m\right)\right\}= \begin{cases}A \backslash\{1, k-a\} & \text { if } i=1, \\ A \backslash\{k-a+1\} & \text { otherwise }\end{cases}
$$

The maximum number of colored $p$ is $\left(\begin{array}{c}k-a-1 \\ m\end{array}\right)$ for any $p$. We can do that because $n>H(a)$. So, $c\left(l_{j}^{i}\right)=c\left(l_{n}^{s}\right), j \neq n$, dan $i \neq s$. Thus, we can arrange such that $\left\{c\left(l_{j t}^{i}\right) \mid t=1,2,3, \ldots, m\right\} \neq$ $\left\{c\left(l_{n t}^{s}\right) \mid t=1,2,3, \ldots, m\right\}$. As the result, by Lemma 2.1, $c$ is a locating coloring. Thus, $\chi_{L}\left(n S_{k, m}\right) \leq$ $k-a+1$ for $n>H(a)$. As the conclusion, we obtain that $\chi_{L}\left(n S_{k, m}\right)=k-a+1$.

For an illustration, we give the locating-chromatic coloring of $n S_{5,3}$ for $1 \leq n \leq 4$ in Figure 1 and $n S_{5,3}$ for $n>4$ in Figure 2 .

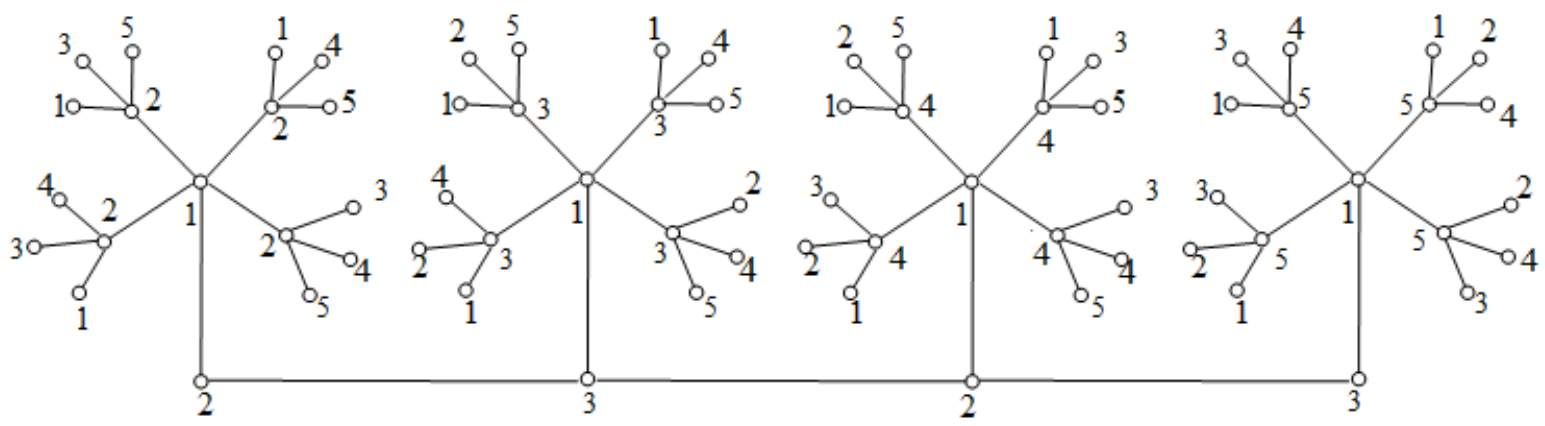

Figure 1. A minimum locating coloring of $4 S_{5,3}$ 


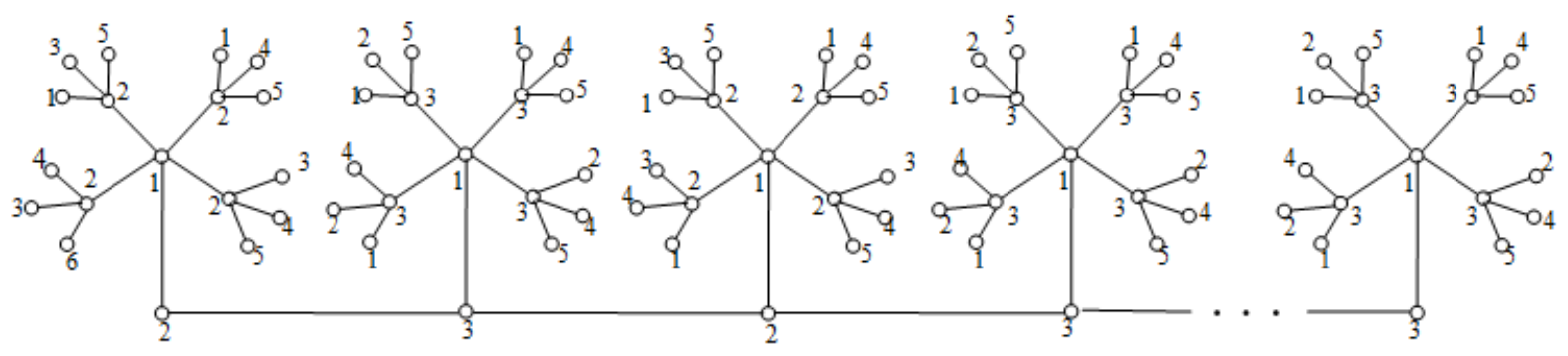

Figure 2. A minimum locating coloring of $n S_{5,3}$ for $n>4, a=0$

\section{References}

[1] Asmiati, H. Assiyatun, E.T. Baskoro, Locating-Chromatic Number of Amalgamation of Stars, ITB J.Sci. 43A (2011), $1-8$.

[2] Asmiati, H. Assiyatun, E.T. Baskoro, D. Suprijanto, R. Simanjuntak, S. Uttunggadewa, Locating-Chromatic Number of Firecracker Graphs, Far East Journal of Mathematical Sciences 63(1) (2012), $11-23$.

[3] Asmiati, E.T. Baskoro, Characterizing of Graphs Containing Cycle with Locating-Chromatic Number Three, AIP Conf. Proc. 1450 (2012), 351 - 357.

[4] Asmiati, Locating-Chromatic Number of Non Homogeneous Amalgamation of Stars, Far East Journal of Mathematical Sciences 93(1) (2014), 89 - 96.

[5] Asmiati, Locating-chromatic number of banana tree, International Mathematical Forum, 12(1) (2017), $39-45$.

[6] Asmiati, Bilangan kromatik lokasi $\mathrm{n}$ amalgamasi bintang yang dihubungkan oleh suatu lintasan, Jurnal Matematika Integratif 13(2) (2017), 115 - 121.

[7] E.T. Baskoro, Asmiati, Characterizing all Trees with Locating-Chromatic Number 3, Electronic Journal of Graph Theory and Applications 1(2) (2013), 109 - 117. 
[8] E.T. Baskoro, I. A. Purwasih, The Locating-Chromatic Number for Corona Product of Graphs, Southeast-Asian J. of Sciences 1(1) (2012), 126 - 136.

[9] G. Chartrand, D. Erwin, M.A. Henning, P.J. Slater, P. Zang , The Locating-Chromatic Number of a Graph, Bulls. Inst. Combin. Appl. 36 (2002), 89 - 101.

[10] G. Chartrand, D. Erwin, M.A. Henning, P.J. Slater, P. Zang, Graph of Order $n$ with LocatingChromatic Number $n-1$, Discrete Mathematics 269 (2003), 65 - 79.

[11] G. Chartrand, P. Zhang, E. Salehi, On the Partition Dimension of Graph, Congr. Numer. 130(1998), $157-168$. 